\title{
Temporal Multi-Looking of SAR Image Series for Glacier Velocity Determination and Speckle Reduction
}

Other Conference Item

Author(s):

Leinss, Silvan (1D; Li, Shiyi; Bernhard, Philipp; Frey, Othmar (1)

Publication date:

2020-05-06

Permanent link:

https://doi.org/10.3929/ethz-b-000453807

Rights / license:

Creative Commons Attribution 4.0 International

Originally published in:

EGUsphere, https://doi.org/10.5194/egusphere-egu2020-3643 
EGU2020-3643

https://doi.org/10.5194/egusphere-egu2020-3643

EGU General Assembly 2020

(c) Author(s) 2020. This work is distributed under

the Creative Commons Attribution 4.0 License.

\title{
Temporal Multi-Looking of SAR Image Series for Glacier Velocity Determination and Speckle Reduction
}

\author{
Silvan Leinss, Shiyi Li, Philipp Bernhard, and Othmar Frey \\ ETH Zürich, Institut für Umweltingenieurwissenschaften (IFU), BAUG, Zürich, Switzerland (leinss@ifu.baug.ethz.ch)
}

The velocity of glaciers is commonly derived by offset tracking using pairwise cross correlation or feature matching of either optical or synthetic aperture radar (SAR) images. SAR images, however, are inherently affected by noise-like radar speckle and require therefore much larger images patches for successful tracking compared to the patch size used with optical data. As a consequence, glacier velocity maps based on SAR offset tracking have a relatively low resolution compared to the nominal resolution of SAR sensors. Moreover, tracking may fail because small features on the glacier surface cannot be detected due to radar speckle. Although radar speckle can be reduced by applying spatial low-pass filters (e.g. $5 \times 5$ boxcar), the spatial smoothing reduces the image resolution roughly by an order of magnitude which strongly reduces the tracking precision. Furthermore, it blurs out small features on the glacier surface, and therefore tracking can also fail unless clear features like large crevasses are visible.

In order to create high resolution velocity maps from SAR images and to generate speckle-free radar images of glaciers, we present a new method that derives the glacier surface velocity field by correlating temporally averaged sub-stacks of a series of SAR images. The key feature of the method is to warp every pixel in each SAR image according to its temporally increasing offset with respect to a reference date. The offset is determined by the glacier velocity which is obtained by maximizing the cross-correlation between the averages of two sub-stacks. Currently, we need to assume that the surface velocity is constant during the acquisition period of the image series but this assumption can be relaxed to a certain extend.

As the method combines the information of multiple images, radar speckle are highly suppressed by temporal multi-looking, therefore the signal-to-noise ratio of the cross-correlation is significantly improved. We found that the method outperforms the pair-wise cross-correlation method for velocity estimation in terms of both the coverage and the resolution of the velocity field. At the same time, very high resolution radar images are obtained and reveal features that are otherwise hidden in radar speckle.

As the reference date, to which the sub-stacks are averaged, can be arbitrarily chosen a smooth flow animation of the glacier surface can be generated based on a limited number of SAR images. The presented method could build a basis for a new generation of tracking methods as the method is excellently suited to exploit the large number of emerging free and globally available high resolution SAR image time series. 
